\title{
Molecular characterisation of congenital glaucoma in a consanguineous Canadian community: a step towards preventing glaucoma related blindness
}

\author{
S Nicole Martin, Joanne Sutherland, Alex V Levin, Robert Klose, Megan Priston, \\ Elise Héon
}

Vision Science Research Program, Toronto Western Hospital Research Institute, University Health Network, 399 Bathurst Street, Room 6-412, Toronto,

Ontario, Canada

M5T 2S8

S N Martin

R Klose

M Priston

E Héon

Department of Ophthalmology, The Hospital for Sick Children, University of Toronto, Toronto,

Canada

J Sutherland

A V Levin

E Héon

The Research Institute, The Hospital for Sick Children,

University of Toronto, Toronto, Canada A V Levin

E Héon

Correspondence to:

Dr Héon,

eheon@playfair.utoronto.ca

Revised version received 3 January 2000

Accepted for publication 14 January 2000

\begin{abstract}
Glaucoma is a leading cause of irreversible blindness in Canada. Congenital glaucoma usually manifests during the first years of life and is characterised by severe visual loss and autosomal recessive inheritance. Two disease loci, on chromosomes $1 \mathrm{p} 36$ and $2 \mathrm{p} 21$, have been associated with various forms of congenital glaucoma. A branch of a large six generation family from a consanguineous Amish community in south western Ontario was affected with congenital glaucoma and was studied by linkage and mutational analysis to identify the glaucoma related genetic defects. Linkage analysis using the MLINK component of the LINKAGE package ( $v$ 5.1) showed evidence of linkage to the $2 \mathrm{p} 21$ region $(Z \max =3.34, \theta=0, D 2 S 1348$ and D2S1346). Mutational analysis of the primary candidate gene, $C Y P 1 B 1$, was done by direct cycle sequencing, dideoxy fingerprinting analysis, and fragment analysis. Two different disease causing mutations in exon 3, 1410del13 and $1505 \mathrm{G} \rightarrow \mathrm{A}$, both segregated with the disease phenotype. The two different combinations of these alleles appeared to result in a variable expressivity of the phenotype. The compound heterozygote appeared to have a milder phenotype when compared to the homozygotes for the 13 bp deletion. The congenital glaucoma phenotype for this large inbred Amish family is the result of mutations in CYP1B1 (2p21). The molecular information derived from this study will be used to help identify carriers of the CYP1B1 mutation in this community and optimise the management of those at risk of developing glaucoma.

(f Med Genet 2000;37:422-427)
\end{abstract}

Keywords: congenital glaucoma; CYP1B1; gene; genetic counselling

Glaucoma constitutes a leading cause of irreversible blindness in Canada. ${ }^{1}$ Congenital and infantile glaucoma are associated with anomalous development of the trabecular meshwork and anterior chamber angle, which leads to an increased resistance to aqueous outflow and raised intraocular pressure (IOP). ${ }^{2}$ Clinical characteristics of congenital glaucoma most often manifest during the first years of life and include tearing, blepharospasm, and photophobia. Corneal oedema, Haab striae (breaks in Descemet membrane), enlarged cornea, optic nerve cupping, and buphthalmos may also develop if the pressure is not controlled. When the diagnosis of congenital glaucoma is delayed, the resulting visual outcome is often poor. ${ }^{34}$ Even though treatments are available, earlier detection is needed in order to prevent or minimise the glaucoma related visual loss.

The primary cause of the disease is unknown. ${ }^{25}$ However, congenital glaucoma is heritable and the majority of cases are autosomal recessive. ${ }^{6}$ Cases of incomplete penetrance are well documented. ${ }^{7}$ The incidence of congenital glaucoma varies depending on geographical location: 1:2500 births in the Middle East, 1:10 000 births in western countries, and 1:1250 births in the Gypsy population of Slovakia. ${ }^{4-10}$

Congenital glaucoma is genetically heterogeneous with two associated loci on chromosomes 1 p36 and 2 p21, respectively. ${ }^{11}{ }^{12}$ Other forms of early onset glaucoma, mapped to chromosomes 6 p25 and 4 q25, ${ }^{13}{ }^{14}$ are usually associated with other congenital anterior segment anomalies which only occasionally result in glaucoma at birth or in infancy and are not considered as "primary congenital glaucoma". $C Y P 1 B 1$, the disease causing gene at the chromosome $2 \mathrm{p} 21$ locus, is proposed to be the predominant congenital glaucoma gene, playing a role in an estimated $85 \%$ of cases. ${ }^{12}$

A large, consanguineous Amish family affected with congenital glaucoma, from south western Ontario, was recruited and studied in an attempt to identify the disease causing genetic defect in that community. In addition, several members of this family were characterised with an atypical form of iris hypoplasia that was studied separately.

\section{Materials and methods}

CLINICAL ASSESSMENT

The project was approved by the Toronto Hospital Human Subjects Review Committee and The Hospital for Sick Children Research Ethics Board. After informed consent, all participants were questioned on their personal medical history and a family tree was drawn. All participants, except for the nuclear family of the proband, were examined in their home where no electricity was available. As a result, no ocular photographs were taken. The proband and his immediate family had a comprehensive eye examination at The Hospital for Sick Children. The affected status was defined by an intraocular pressure greater than $25 \mathrm{~mm}$ $\mathrm{Hg}$ in the first years of life and by the presence 
of corneal enlargement, scar, or Haab striae or a history consistent with congenital glaucoma. As the enlargement of the eye (buphthalmos) and cornea can only occur in the first few years of life, people with buphthalmos in their teens and adulthood are presumed to have a form of congenital or infantile glaucoma. Eye charts of older affected people were reviewed. Affected subjects and their relatives were examined with a portable slit lamp and direct ophthalmoscopy. The charts from their respective eye physicians were reviewed when available.

\section{GENOTYPING}

DNA was prepared from whole blood (10-20 $\mathrm{ml}$ ) using a non-organic procedure. ${ }^{15}$ The selection of short tandem repeat polymorphisms (STRPs) was done using genetic map information from publications and genome databases, Généthon, CHLC, and Marshfield. Primers were obtained from Research Genetics Inc (screening set 6A) or from ACGT Inc (Toronto, ON). A fluorescent dye label was incorporated on the 5' end of one of the primers and the protocol used for genotyping was previously described. ${ }^{16}$ The genotyping was done blinded to the affected status.

LINKAGE ANALYSIS OF CONGENITAL GLAUCOMA Four STRP markers that mapped to the region $2 \mathrm{p} 21$, around $C Y P 1 B 1$, were used and their telomeric to centromeric order with intermarker distances (in $\mathrm{cM}$ ) were as follows: D2S1788 - (3) - D2S177 - D2S1348 D2S1346. Linkage analysis was performed with the MLINK component of the LINKAGE package (v 5.1). Lod scores were obtained with the assumption of an autosomal recessive mode of inheritance, full penetrance, a disease gene frequency of 0.0001 , and equal allele frequencies.

LINKAGE ANALYSIS OF IRIS HYPOPLASIA

Molecular characterisation of the iris hypoplasia variant observed in some family members was done by linkage analysis using additional markers covering the three previously reported loci on chromosomes 4q25, 6p25, and 13q14. ${ }^{1317}$ The markers used for each loci including the intermarker distance (in $\mathrm{cM}$ ) are as follows: 4q25 (D4S3240 - D4S2623 - (3) D4S406 - (4.5) - D4S2392), 6p25 (D6S1600 (1.4) - D6S967 - D6S344 - (6.9) - D6S477), and 13p14 (D13S1493 - (7.5) - D13S894 (1.65) - D13S1253 - (3.8) - D13S263 - (6.3) D13S788).

MUTATIONAL ANALYSIS OF CYP1B1

CYP1B1 consists of three exons, only two of which code for the protein. PCR primers used for the mutational analysis of exons 2 and 3 of the gene CYP1B1 were previously described. $^{719}$ Additional exon 3 primers were designed from the mRNA sequence (Acc No U56438) (5'catgattcacagaccactgg3'-reverse and 5'ccagctcgattcttggacaa3'-forward). ${ }^{20}$

SEQUENCING OF CYP1B1

Mutational analysis of $C Y P 1 B 1$ used direct sequencing from genomic DNA. Methods of
PCR amplification were as previously described. ${ }^{21}$ Gene specific primers tailed with M13 universal primer 5'gtaaaacgacggccagt3' or M13 reverse primer 5'cacaggaaacagctatgac3' were used. The amplicon was purified using QIAquick PCR Purification $\mathrm{Kit}^{\mathrm{TM}}$ (Qiagen, Mississauga) according to the manufacturer's protocol. The column purified amplicon was then sequenced on a MicroGene Blaster ${ }^{\mathrm{TM}}$ automated DNA sequencing unit (Visible Genetic Inc (VGI), Toronto) using Cy5.5 labelled M13 universal or M13 reverse primers and the Thermo Sequenase ${ }^{\mathrm{TM}}$ Cycle Sequencing Core Kit (US 79610, VGI) as previously published. ${ }^{21}$

\section{FRAGMENT ANALYSIS OF CYP1B1}

Family members were screened for the $13 \mathrm{bp}$ deletion by fragment analysis as follows. M13 tailed primers were used to amplify a $320 \mathrm{bp}$ fragment in the 5 ' region of exon 3 . Then $1 \mu \mathrm{l}$ of unpurified amplicon, $1.5 \mathrm{mmol} / 1 \mathrm{MgCl}_{2}, 1 \times$ PCR buffer II, $2 \mathrm{mmol} / \mathrm{l}$ of each dNTP, and 0.75 pmol of M13 universal primer (CY5.5 labelled) and the unlabelled reverse primer in the initial amplification were used in a second PCR reaction. The PCR reaction used the following conditions: $94^{\circ} \mathrm{C}$ for three minutes, $\left(94^{\circ} \mathrm{C}\right.$ for 30 seconds, $51^{\circ} \mathrm{C}$ for 30 seconds, $70^{\circ} \mathrm{C}$ for five seconds) $\times 28$ cycles and $70^{\circ} \mathrm{C}$ for an eight minute extension. Formamide loading dye $(1.5 \mu \mathrm{l})$ was mixed with $1.5 \mu \mathrm{l}$ of the final amplified product, denatured for one minute, and then electrophoresed on a $6 \%$ Surefill $^{\mathrm{TM}}$ sequencing gel (VGI, Toronto). Two control size markers were run with each sample.

DIDEOXY FINGERPRINTING ANALYSIS OF CYP1B1 Screening for the $1505 \mathrm{G} \rightarrow \mathrm{A}$ missense mutation was done by dideoxythymidine fingerprinting analysis. A cycle sequencing reaction was performed with only the $\mathrm{T}$ termination mix from Thermo Sequenase ${ }^{\mathrm{TM}}$ Cycle Sequencing Core Kit and the M13 reverse primer. The conditions for the cycle sequencing reaction are the same as described above.

\section{PROTEIN MODELLING}

Homology modelling was performed using Swiss-Model version 2.0. The reference structure used was P450BM-P which is the best model for eukaryotic P450s. ${ }^{19}{ }^{22}$ The predicted structure for CYP1B1 was viewed in the WEBLAB VIEWERTM PRO (Molecular Simulations Inc, San Diego).

\section{Results}

CLINICAL ANALYSIS

Six bilaterally affected people and 38 unaffected people were recruited. The penetrance appeared complete and the pedigree (fig 1) was consistent with autosomal recessive inheritance. However, the severity of the phenotype appeared to be variable between family members. Buphthalmos and increased intraocular pressure in the first year of life characterised the affected status of the sixth generation, whereas the fourth generation appeared to have an overall milder course. No specific anterior 


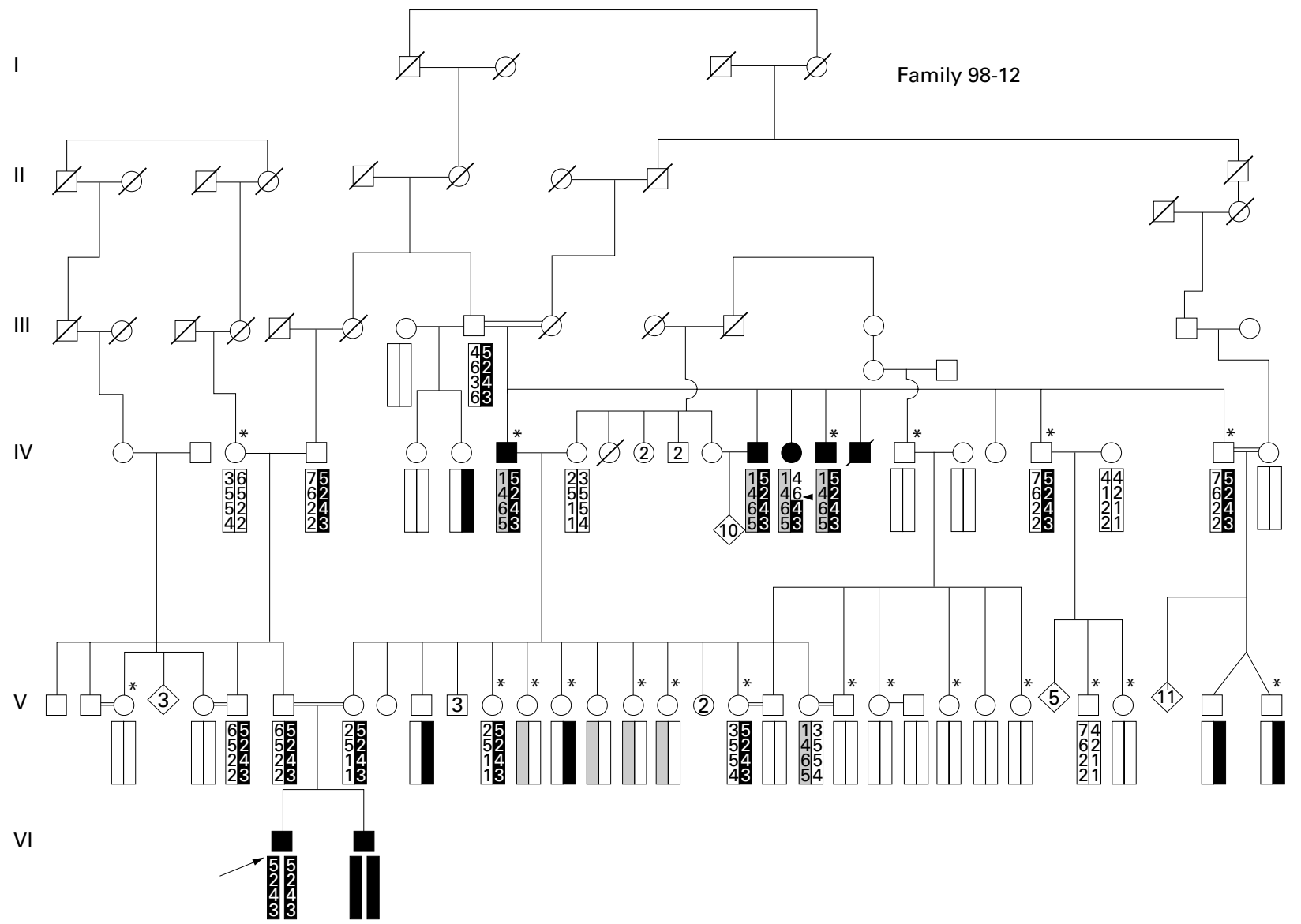

Figure 1 Haplotype analysis and segregation analysis of CYP1B1 mutant alleles. Pedigree with haplotypes for selected markers on chromosome $2 p 21$ markers (marker order:pter, D2S1788, D2S177, D2S1348, D2S1346, cen). Blackened symbols denote subjects affected with congenital glaucoma while the asterisk indicates those affected with iris hypoplasia. The boxed haplotypes segregate with the affected status. The black boxes indicate the 1410 del13 mutant allele, the grey boxes the $1505 G \rightarrow A$ mutant allele, and the white boxes indicate a normal allele as determined by mutational analysis. IV.14 showed a single recombination placing the disease locus centromeric to D2S177. The hatched boxes show the segregation of the 1410 del13 mutant allele while the grey boxes show the segregation of the $1505 G \rightarrow A$ mutant allele.

segment malformation was noted. The clinical information is summarised in table 1 and detailed as follows.

VI.1 (4 years old) presented in the first week of life with corneal oedema and the typical high iris insertion seen in congenital glaucoma, but normal corneal diameters $(11.25 \mathrm{~mm})$. The optic nerve could not be seen at presentation. His glaucoma has been poorly controlled despite maximum medical and surgical treatments. At 4 years of age he required bilateral laser cyclophotoablation (destruction of the ciliary body) and still continues on multiple topical antiglaucoma medications. His course has been complicated by a severe cone-rod retinal dystrophy confirmed by electroretinography, developmental delay, and failure to thrive. Karyotype and extensive investigations to characterise the nature of his nonophthalmic findings have been unrevealing. His current vision is at best hand motion and felt largely to be because of the retinal dystrophy. The right optic nerve cup is approximately 0.3 but is difficult to assess. Corneal diameters are now enlarged, $14.0 \mathrm{~mm}$ OD and $14.25 \mathrm{~mm}$ OS.

Table 1 Clinical characteristics of subjects with congenital glaucoma

\begin{tabular}{|c|c|c|c|c|c|c|}
\hline Case & $\begin{array}{l}\text { Age at } \\
\text { diagnosis }\end{array}$ & $\begin{array}{l}\text { Corneal diameter at diagnosis } \\
O D ; O S\end{array}$ & $\begin{array}{l}\text { IOP at diagnosis } \\
\text { OD;OS }\end{array}$ & $\begin{array}{l}\text { Last vision } O D ; O S \\
\text { (age) }\end{array}$ & $\begin{array}{l}\text { Last } C / D \text { ratio } \\
\text { (age) OD;OS }\end{array}$ & Treatments \\
\hline VI.1 & $<1 \mathrm{wk}$ & $11.25 \mathrm{OU}$ (normal) & $18 ; 21(\mathrm{~mm} \mathrm{Hg})$ & LP; HM & $0.3 ; \mathrm{N} / \mathrm{A}$ & $\begin{array}{l}\text { Medical treatment, surgery } \times 3 \text {, laser } \\
\text { surgery }\end{array}$ \\
\hline VI. 2 & $<1 \mathrm{wk}$ & $12 \mathrm{~mm} ; 12.75$ (enlarged OS) & $22 ; 49(\mathrm{~mm} \mathrm{Hg})$ & $\begin{array}{l}\text { Amblyopia OD } \\
\text { CSM OS }\end{array}$ & $0.1 \mathrm{OU}$ & $\begin{array}{l}\text { Medical treatment, surgery } \times 3 \text {, laser } \\
\text { surgery }\end{array}$ \\
\hline IV.7 & $\sim 14 \mathrm{y}$ & Enlarged OD & Raised OU & $20 / 200 ; 20 / 15(53$ y) & $0.9 ; 0.1$ & $\begin{array}{l}\text { 1957-73: medical treatment OU } \\
\text { 1973: medical and surgical treatment }\end{array}$ \\
\hline IV.13 & $\sim 14 \mathrm{y}$ & Enlarged OU & Not available & 20/15 OD teenage & None & Medical treatment OU/ trauma OS in 1969 \\
\hline IV. 14 & Birth & $\begin{array}{l}\text { Enlarged OU } \\
\text { Buphthalmos OU }\end{array}$ & Raised OU & Enucleated $\mathrm{OU}$ & Enucleated OU & Enucleation at $2 \mathrm{y}$, traumatic cause? \\
\hline IV.15 & $\sim 14 \mathrm{y}$ & Buphthalmos OU & $37 \mathrm{~mm} \mathrm{Hg}$ OU & Not available & Pale nerve OU & $\begin{array}{l}\text { 1955-67: medical treatment, 1968: medical } \\
\text { and surgical treatment }\end{array}$ \\
\hline
\end{tabular}

OD: right eye, OS: left eye, OU: both eyes

$\mathrm{C} / \mathrm{D}$ : cup disc ratio of the optic nerve

IOP: intraocular pressure.

LP: light perception, HM: hand motion.

CSM: central, steady and maintained. 
VI.2 (5 years old) also presented in the first weeks of life with cloudy, enlarged corneas and findings consistent with congenital glaucoma. His condition was also difficult to control despite maximum medical and surgical interventions and laser cyclophotoablation. His corneal diameters are now $14.25 \mathrm{~mm}$ OD and $14.00 \mathrm{~mm}$ OS. Optic nerve cuppings are 0.1 OU and pressures are well controlled on medical therapy.

IV.7 (56 years old) was diagnosed at 14 years with right eye (OD) buphthalmos and raised pressures in both eyes (OU). His intraocular pressures were controlled medically until the age of 30, when the pressure rose to $36 \mathrm{mmHg}$ and the vision OD decreased to 20/200. Surgery of the right eye was required to control the disease which is currently stabilised with the addition of medical therapy.

IV.13 (63 years old) had bilateral buphthalmos diagnosed in his late teenage years but retained a good central vision OD with medical treatment until 1997 when he was last seen. $\mathrm{He}$ suffered traumatic visual loss OS in 1969.

IV.14 (61 years old) was born with glaucoma and bilaterally enucleated at 2 years of age. It is unclear if the enucleation was related to glaucoma or to a severe trauma she suffered in early childhood.

IV.15 ( 58 years old) was diagnosed at the age of 14 years with bilateral buphthalmos, mild optic nerve pallor, and intraocular pressures of $37 \mathrm{~mm} \mathrm{Hg}$ in both eyes. $\mathrm{He}$ was controlled medically until 1967 when surgery was required to control the pressure in both his eyes. He has remained stable for the last 30 years with sporadic use of his glaucoma drops.

Twenty people were also affected with a type of iris hypoplasia ( ${ }^{\star}$ in fig 1 ) that did not segregate with the congenital glaucoma phenotype. The absence of iris colarette and a variable degree of thinned anterior leaflet of the iris from the pupillary border to the iris base with the iris base being the thinnest characterised this phenotype. The stromal strands had a tight appearance and the pigment epithelium of the iris could be seen through the strands to a variable degree. These people were otherwise normal; no transillumination defects were observed, nor were the classical features of Rieger syndrome. ${ }^{23}$ The iris hypoplasia appeared to have an autosomal dominant mode of inheritance.

LINKAGE ANALYSIS OF CONGENITAL GLAUCOMA A subset of the branch of the family was genotyped and studied by linkage analysis (fig 1). This included five affected subjects and 14 unaffected subjects. Affected subject VI.2 was recruited after linkage analysis was completed.

Table 2 Two point linkage data

\begin{tabular}{|c|c|c|c|c|c|c|c|c|c|}
\hline \multirow[b]{2}{*}{ Marker } & \multirow{2}{*}{$\begin{array}{l}I M D \\
(c M)\end{array}$} & \multicolumn{6}{|c|}{ Lod score at $\theta=$} & \multirow[b]{2}{*}{$\theta \max$} & \multirow[b]{2}{*}{$Z \max$} \\
\hline & & 0.00 & 0.05 & 0.10 & 0.20 & 0.30 & 0.40 & & \\
\hline D2S1788 & & $-\infty$ & 1.74 & 1.71 & 1.34 & 0.84 & 0.35 & 0.066 & 1.76 \\
\hline D2S177 & 3 & $-\infty$ & 1.52 & 1.51 & 1.19 & 0.74 & 0.30 & 0.071 & 1.54 \\
\hline D2S1346 & 0 & 3.34 & 3.0 & 2.64 & 1.91 & 1.16 & 0.44 & 0.000 & 3.34 \\
\hline D2S1348 & 0 & 3.34 & 3.0 & 2.64 & 1.91 & 1.16 & 0.44 & 0.000 & 3.34 \\
\hline
\end{tabular}

IMD: intermarker distance.
The first candidate locus was the CYP1B1 locus on chromosome $2 \mathrm{p} 21$ because of its suggested role in congenital glaucoma. Two point linkage data for the four STRP markers are summarised in table 2. Evidence for linkage to the locus at $2 \mathrm{p} 21$ was obtained with markers $\mathrm{D} 2 \mathrm{~S} 1348$ and $\mathrm{D} 2 \mathrm{~S} 1346 \quad(\mathrm{Zmax}=3.34$, $\theta \max =0)$.

Haplotype analysis showed that the affected haplotypes segregated perfectly with the congenital glaucoma phenotype in this family (fig 1). A recombination event observed in affected subject IV.14 places the candidate gene CYP1B1 centromeric to D2S177. This recombination event also clarifies the order of the two previously non-recombinant markers, D2S177 and D2S1346 (fig 1).

LINKAGE ANALYSIS OF IRIS HYPOPLASIA

Two point linkage data for the iris hypoplasia phenotype at loci 4q25, 6p25, and 13q14 failed to identify linkage to these loci. Lod score values lower then -2 were obtained for several markers at each locus (data not shown).

MUTATIONAL ANALYSIS OF CYP1B1

$C Y P 1 B 1$ was the primary candidate gene to study. The two coding exons were directly sequenced in one affected person (IV.7, fig 1) and two mutations within exon 3 were identified (data not shown). One mutation is a $13 \mathrm{bp}$ deletion of nucleotides 1410-1422 (1410del13), which creates a frameshift premature stop codon, and truncation of the protein (Acc No U56438). ${ }^{20}$ The other mutation was a missense mutation, $1505 \mathrm{G} \rightarrow \mathrm{A}$, substituting the highly conserved amino acid glutamic acid for lysine (Glu387Lys, Acc No 3913312 ). These mutations were previously reported separately, as was the screening of a randomly selected control population. Neither mutation was present in 200 control chromosomes. ${ }^{12} 19$

Screening of the other 43 family members using fragment analysis and dideoxythymidine fingerprinting confirmed that both mutations cosegregated with the disease phenotype (fig 1). Segregation of the mutant alleles was consistent with an autosomal recessive mode of inheritance. Mutational analysis identified four affected subjects who were compound heterozygotes, two affected subjects who were homozygous for the $13 \mathrm{bp}$ deletion, and 19 subjects who were carriers of either mutation (fig 1). The congenital glaucoma phenotypes associated with the different combination of mutations showed variable degrees of severity. Homozygotes for the 1410 del13 appeared to have a more severe phenotype than those who were compound heterozygotes for the two described mutations. Unfortunately, there is missing clinical information for IV.14, leaving unclear the severity of her disease.

No correlations were observed between the $C Y P 1 B 1$ genotypes and the iris hypoplasia phenotype confirming that the iris hypoplasia observed in this family is unrelated to the congenital glaucoma. There was no evidence of linkage of the iris hypoplasia phenotype to the previously documented loci (data not shown), 
supporting the genetic heterogeneity of this condition and suggesting the possibility of an additional iris hypoplasia locus. No person with iris hypoplasia had congenital glaucoma with the exception of IV.7 and IV.13.

\section{Discussion}

Linkage and haplotype analysis identified the disease causing locus on chromosome $2 \mathrm{p} 21$ with $C Y P 1 B 1$ being the disease causing gene for the glaucoma segregating in this Amish family. CYP1B1 encodes for a cytochrome P450 enzyme that belongs to the multigene superfamily of monomeric mixed function mono-oxygenases responsible for phase 1 metabolism of numerous structurally diverse substrates. It is postulated that the oxygenation of a CYP1B1 substrate, still unknown in the eye, would allow the proper functioning of signal transduction pathways involved in eye growth and differentiation. ${ }^{12}$

By directly sequencing $C Y P 1 B 1$ in this family, two mutations in exon 3 were identified. The 1410del13 mutation introduces a stop codon $203 \mathrm{bp}$ downstream of the deletion. The truncation of the protein (amino acids 422-453) eliminates the carboxy-terminus end, which includes the essential haem domain. This may have a deleterious effect on the function of the protein (data not shown). ${ }^{22-25}$ This mutation is suggested to create a functional null allele. ${ }^{6}$ The other mutation altered amino acid 387 from glutamic acid to lysine. Glu387 is located in helix K, which is one of the highly conserved core structural elements. ${ }^{19}$ The core structural elements are suspected to be involved in proper folding of the protein and in active haem binding. ${ }^{192}$ Glu387 is also conserved across all documented species and different P450 enzymes and is one of three absolutely conserved residues identified in P450s. ${ }^{22}$ This conservation of the amino acid supports its importance and suggests that this mutation is likely to affect the proper function of the protein.

The phenotypes between the two affected generations show some differences in severity. Children who were homozygous for the deletion appeared to be more severely affected than those in generation IV who were compound heterozygotes, with perhaps the exception of IV.14. This is not surprising since an important part of this protein is eliminated by the 1410del13 mutation. This homozygous deletion was previously reported but no phenotype was described, ${ }^{12}$ whereas a case homozygous for the $1505 \mathrm{G} \rightarrow \mathrm{A}$ mutation was previously reported with a severe phenotype. ${ }^{8}$ One could speculate that any homozygous impairment of the haem binding domain could lead to a severe phenotype. This combination of the mutations described has not been previously reported and this is the first report of the respective genotype-phenotype correlation. As more mutations are identified, the definition of phenotype-genotype correlation may help to improve the management.

All 44 members of the Amish family were screened for both mutations and the carriers of the disease were identified along with couples whose future children may be at risk of devel- oping glaucoma. Identifying two mutations suggests that there are two distinct founders for the glaucoma segregating in this family. The mutant allele containing the $13 \mathrm{bp}$ deletion is found in both branches of this family increasing the risk of the development of congenital glaucoma in future children considering the high degree of "intracommunity" relationships (not all illustrated in the pedigree shown). The information has been returned to the community to improve genetic counselling and management. The opportunity has also been given to other members of the Amish community to assess their carrier status and to proceed with early screening of their children at risk. Although genetic counselling may be less likely to alter mating patterns of this highly consanguineous population, early identification of at risk couples will hopefully improve the management and outcome of offspring and provide an opportunity to prevent or minimise glaucoma related visual loss.

Data access: Généthon: www.genethon.fr/, Marshfield: www.marshmed.org/genetics/, CHLC: www.chlc.org/, Molecular Simulation Inc: www.msi.com, SWISS-MODEL version 2.0: www.expasy.ch/swissmod/SWISS-MODEL. html, Genbank: www.ncbi.nlm.nih.gov/Web/Search/index.html. The authors are grateful to Gail Billingsley MSc for her constructive discussions, to the families for their enthusiastic participation, to Dr J R Walker who kindly provided medical records, and to Dr V Siu, Dr D Williams-Lyn and Ms E Perruzza for their support in the organisation of this project. This work was funded by the Glaucoma Research Society of Canada, the Weston Foundation, PSI grant No 97-02, Fight For Sight, and the Canadian Genetic Disease Network.

1 Friedman J, Walter M. Glaucoma genetics, present and future. Clin Genet 1999;55:71-9.

2 DeLuise V, Anderson D. Primary infantile glaucoma (congenital glaucoma). Surv Ophthalmol 1983;28:1-19.

3 Shaffer R. Genetics and the congenital glaucoma. Trans Am Acad Ophthalmol Otol 1965;69:253-68.

4 Francois J. Congenital glaucoma and its inheritance. Ophthalmologica 1980;181:61-73.

5 Jerndal T. Congenital glaucoma due to dominant goniodysgenesis. A new concept of the heredity of glaucoma. Am $\mathcal{F}$ Hum Genet 1983;35:645-51.

6 Sarfarazi $M$. Recent advances in molecular genetics of glaucomas. Hum Mol Genet 1997;6:1667-77.

7 Bejiani B, Lewis R, Tomey K, et al. Mutations in CYP1B1, the gene for cytochrome P450B1, are the predominant cause of primary congenital glaucoma in Saudi Arabia. $A m$ f Hum Genet 1998;62:325-33.

8 Plasilova M, Ferakova E, Kadasi L, et al. Linkage of Plasilova $\mathrm{M}$, Ferakova $\mathrm{E}$, Kadasi $\mathrm{L}$, et al. Linkage of
autosomal recessive primary congenital glaucoma to the GLC3A locus in Roms (gypsies) from Slovakia. Hum Hered 1998;48:30-3.

9 Gencik A, Gencikova A, Ferak V. Population genetical aspects of primary congenital glaucoma. I. Incidence, prevalence, gene frequency, and age of onset. Hum Genet 1982;61:193-7.

10 Jaafar $M$. Care of the infantile glaucoma patient. In: Reinecke RD, ed. Ophthalmology annual 1988. New York: Raven Press, 1988:15

11 Akarsu A, Turacli M, Aktan S, et al. A second locus (GLC3B) for primary congenital glaucoma (buphthalmos) maps to the 1 p36 region. Hum Mol Genet 1996;5:1199203.

12 Stoilov I, An A, Sarfarazi M. Identification of three truncating mutations in cytochrome P4501B1 (CYP1B1) as the principal cause of primary congenial glaucoma (buphthalmos) in families linked to the GLC3A locus on chromosome 2p21. Hum Mol Genet 1997;6:641-7.

13 Semina E, Reiter R, Leysens N, et al. Cloning and characterization of a novel bicoid-related homeobox transcription factor gene, REIG, involved in Rieger syndrome. Nat Genet 1996;14:392-9.

4 Nishimura D, Swiderski R, Alward W, et al. The forkhead transcription factor gene FKHL7 is responsible for glaucoma phenotypes which map to 6p25. Nat Genet 1998 ; 19:140-7

15 Miller S, Dykes D, Polesky H. A simple salting out procedure for extracting DNA from human nucleated cells. Nucleic Acids Res 1988;16:1215.

16 Heon E, Liu S, Billingsley G, et al. Gene localization for aculeiform cataract, on chromosome 2q33-35. Am f Hum Genet 1998;63:921-6.

17 Mears A, Farideh M, Gould D, Pearce W, Walter M. Autosomal dominant iridogoniodysgenesis anomaly maps to 6p25. Am ₹ Hum Genet 1996;59:1321-7. 
18 Phillips J, Del Bono E, Haines J, et al. A second locus for Rieger syndrome maps to chromosome 13q14. Am f Hum

19 Stoilov I, Akarsu A, Alozie I, et al. Sequence analysis and homology modeling suggest that primary congenital glaucoma on 2 p21 results from mutations disrupting either the hinge region or the conserved core structures of
cytochrome P4501B1. Am f Hum Genet 1998;62:573-84. 20 Sutter T, Tang Y, Hayes C, et al. Complete cDNA sequence of a human dioxin-inducible mRNA identifies a new gene subfamily of cytochrome $\mathrm{P} 450$ that maps to chromosome 2. F Biol Chem 1994;269:13092-9.

21 Heon E, Priston M, Schorderet D, et al. The gamma crystal- lins and human cataracts: a puzzle made clearer. Am f Hum Genet 1999;65:1261-7.

22 Graham-Lorence S, Peterson J. Structural alignments of 450s and extrapolations to the unknown. Methods Enzymol 1996;272:315-27.

23 Shields M, Buckley E, Klintworth G, Thresher R. AxenfeldRieger syndrome. A spectrum of developmental disorders. Surv Ophthalmol 1985;29:387-409.

24 Guengerich F. Mini review. Cytochrome P-450. Comp Biochem Physiol 1988;89C:1-4.

25 Gonzalez F. The molecular biology of cytochrome P450s. Pharmacol Rev 1989;40:443-288. 